\title{
Noncommutative strong maximals and almost uniform convergence in several directions - Addendum
}

\author{
José M. Conde-Alonso ${ }^{1}$, Adrián M. González-Pérez ${ }^{2}$ and Javier Parcet ${ }^{3}$ \\ ${ }^{1}$ UAM - Departamento de Matemáticas, 7 Francisco Tomás y Valiente, 28049 Madrid, Spain; E-mail: jose.conde@uam.es. \\ ${ }^{2}$ IMPAN (Instytut Matematyczny Polskiej Akademii Nauk), ul. Sniadekich 8, 00-656 Warsaw; \\ E-mail: agonzalez-perez@impan.pl. \\ ${ }^{3}$ Consejo Superior de Investigaciones Científicas - ICMAT, 23 Nicolás Cabrera, 28049 Madrid, Spain; \\ E-mail: parcet@icmat.es.
}

The original publication of this article was published without an important acknowledgement, which is given here:

The first author is supported by the Madrid Government (Comunidad de Madrid-Spain) under the Multiannual Agreement with Universidad Autonoma de Madrid in the line of action encouraging youth research doctors, in the context of the V PRICIT, grant SII/PJI/2019-00514.

\section{References}

J. Conde-Alonso, A. González-Pérez and J. Parcet, 'Noncommutative strong maximals and almost uniform convergence in several directions', Forum Math. Sigma 8 (2020), E57. doi:10.1017/fms.2020.37.

\footnotetext{
(c) The Author(s), 2021. Published by Cambridge University Press. This is an Open Access article, distributed under the terms of the Creative Commons Attribution licence (http://creativecommons.org/licenses/by/4.0/), which permits unrestricted re-use, distribution, and reproduction in any medium, provided the original work is properly cited.
} 